\title{
Self-Trapping of Magnetic Oscillation Modes in Landau Flux-Closure Structures
}

\author{
A. Krasyuk, F. Wegelin, S. A. Nepijko, H. J. Elmers, ${ }^{*}$ and G. Schönhense \\ Institut für Physik, Universität Mainz, D-55128 Mainz, Germany \\ M. Bolte \\ Institut für Angewandte Physik und Zentrum für Mikrostrukturforschung, Universität Hamburg, Jungiusstrasse 11, \\ Hamburg D-20355, Germany \\ C. M. Schneider \\ Institut für Festkörperforschung IFF-6, Forschungszentrum Jülich GmbH, D-52425 Jülich, Germany
}

(Received 29 July 2005; published 7 November 2005)

\begin{abstract}
We investigated the magnetodynamics in rectangular Permalloy platelets by means of time-resolved $\mathrm{x}$-ray photoemission microscopy. $10 \mathrm{~nm}$ thick platelets of $16 \times 32 \mu \mathrm{m}$ size were excited by an oscillatory field along the short side of the sample with a fundamental frequency of $500 \mathrm{MHz}$ and considerable contributions of higher harmonics. Under the influence of the oscillatory field, the Néel wall in the initial classical Landau pattern shifts away from the center, corresponding to an induced magnetic moment perpendicular to the exciting field. This phenomenon is explained by a self-trapping effect of the dominating spin-wave mode when the system is excited just below the resonance frequency. The basic driving mechanism is the maximization of entropy.
\end{abstract}

DOI: 10.1103/PhysRevLett.95.207201

PACS numbers: $75.40 . \mathrm{Gb}, 75.60 .-\mathrm{d}, 75.75 .+\mathrm{a}$

Excitations of magnetic moments in nanostructures have recently attracted considerable attention [1-5] due to their relevance to high-frequency applications of magnetic particles in data storage devices. Advanced magnetic recording technology pushes the switching time into the gyromagnetic regime. For mesoscopic elements, the high-frequency behavior is governed by confined spinwave eigenmodes as visible, e.g., in Brillouin light scattering $[2,6]$. So far, most studies have addressed the simplest case of elements in an almost monodomain state [6,7]. In larger microstructures, magnetic stray fields are minimized by the formation of multidomain configurations. Thin rectangular platelets of Permalloy often exhibit a Landau flux-closure structure comprising a Néel wall in the center [8].

The magnetization dynamics is quantitatively described by the Landau-Lifshitz-Gilbert equation [9] that resembles the equation of motion for a spinning top, because the magnetic moment is accompanied by an angular momentum. In the ultrafast regime, the torque acting on the local magnetization $\vec{M}$ becomes the dominant factor. This torque may initiate a precessional motion of $\vec{M}$, which can be observed, if the Fourier spectrum of the excitation by an external field comprises significant components of the precessional eigenfrequency of the system. In particular, $\vec{M}$ should not be affected, if the local torque disappears.

In this Letter, we report on the striking phenomenon of a magnetic moment induced perpendicular to an exciting ac magnetic field. This phenomenon occurs for a Landau flux-closure structure excited slightly off resonance. We show that the induced perpendicular moment, which leads to a domain wall shift despite zero local torque, is caused by a self-trapping of an oscillating mode, thus maximizing the energy exhausted off the exciting field.

The time dependent spatial distribution of the magnetization was measured using a photoemission electron microscope (PEEM) [10]. The PEEM measures the spatial distribution of the $\mathrm{x}$-ray absorption via the electron yield of secondary electrons [11]. When the energy of circularly polarized (polarization vector $\vec{P}$ ) photons is tuned to the $\mathrm{Ni}-L_{3}$ absorption edge, the electron yield varies with the relative orientation of magnetization $(\vec{M})$ and $\vec{P}$. X-ray magnetic circular dichroism (XMCD) images are obtained from two images taken with opposite polarization $(+$ and $-)$. In order to visualize magnetic domains, we calculated the asymmetry $I_{i}=\left(I_{i}^{+}-I_{i}^{-}\right) /\left(I_{i}^{+}+I_{i}^{-}\right)$at each pixel (Fig. 2), which is proportional to $\vec{M} \cdot \vec{P}$. The samples are Permalloy $\left(\mathrm{Ni}_{80} \mathrm{Fe}_{20}\right)$ platelets with $16 \mu \mathrm{m}$ width, $32 \mu \mathrm{m}$ length, and $10 \mathrm{~nm}$ thickness, placed on a coplanar waveguide (Cu, $50 \mu \mathrm{m}$ width, $200 \mathrm{~nm}$ thickness) with the long side parallel to the waveguide. The in-plane direction of the $\mathrm{x}$-ray beam (incident angle $65^{\circ}$ ) was oriented perpendicular to the waveguide and parallel to the exciting field. Because of the grazing incidence of the $\mathrm{x}$ rays, we are most sensitive to the in-plane magnetization component along the short side of the platelet.

An externally triggered pulse generator injected the high-frequency current pulses into the waveguide. The actual pulse shape [see curves I, II, and III in Fig. 1(a)] was derived from the apparent change of the sample size, when the current pulse passes the field of view. The voltage pulse accompanying the current pulse slightly changes the magnification of the electron optical lens [12]. An absolute value for the magnetic field results from the voltage signal measured at the output of the waveguide using a fast 
oscilloscope. The maximum field values are of the order of 2 Oe [Fig. 1(a)]. The output shield of the pulse generator was set to floating ground. Thus, the mean current through the waveguide remains zero, and we merely apply a $500 \mathrm{MHz}$ ac signal with considerable contribution of higher harmonical components [Fig. 1(b)] and synchronized with the x-ray pulses from the synchrotron.

Time-resolved experiments are performed by stroboscopic illumination of the sample by x-ray pulses produced by electron bunches in the synchrotron ring $\left(t_{\mathrm{FWHM}}=\right.$ 3 ps, low $\alpha$ mode [13]) with a repetition rate of $500 \mathrm{MHz}$. The ac driving current and the probe pulses are synchronized via a variable electronic delay $t$. The sample is thus excited and probed every $2 \mathrm{~ns}$. To acquire an image, we typically integrate the signal for $30 \mathrm{~s}$, thus averaging over $1.5 \times 10^{10}$ pump-probe cycles.

Snapshots of the time evolution of the magnetization $\vec{M}$ in the Permalloy platelet comprising a Landau flux-closure pattern are shown in Fig. 2 for the smallest field pulse. Micromagnetic simulations [14] were used to verify the experimental findings. Permalloy rectangles of the same aspect ratio were excited with periodic pulses of the same magnetic pulse shapes and strengths as the experimental samples [see Fig. 1(a)]. The time evolution of the magnetization was stored, until the oscillations converged. The spin-wave eigenfrequencies were then determined by local Fourier transformations, and the local power density was integrated over the volume to a global power density. The local power density yields the location and relative phases of specific eigenmodes.

The domains oriented parallel and antiparallel to $\vec{P}$ appear black and white, while the two domains oriented perpendicular to $\vec{P}$ both appear gray. A $180^{\circ}$-Néel wall along the $y$ axis separates the two large domains with $\vec{M}$ upward (left) and downward (right). In the Néel wall, $\vec{M}$ is oriented to the left; thus, the Néel wall appears black. Because of the high driving frequency, the image at $t=0$

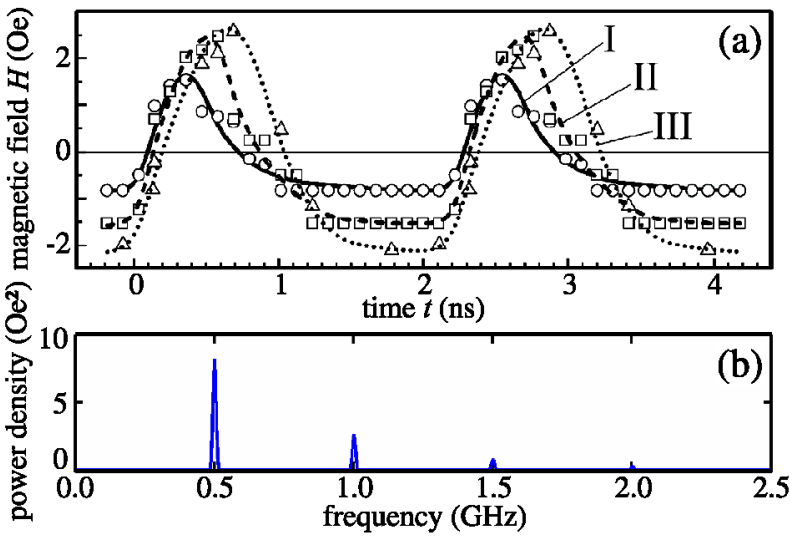

FIG. 1 (color online). (a) Time dependence of the exciting magnetic field $H_{x}(t)$. Two periods are shown in order to emphasize the repetition rate. (b) Fourier transformation of 50 periods of amplitude I into frequency space. corresponding to the onset of the field pulse does not represent the equilibrium case before the excitation. The system does not have enough time to relax back into the equilibrium state before the next pulse arrives. Instead, the image shows already a dynamical state of the magnetization pattern.

At $t=600 \mathrm{ps}$, the intensity has increased in the left and right domains, indicating a rotation of the magnetization vector towards the direction of the applied field, i.e., clockwise in the left domain and counterclockwise in the right domain. At $t=1100 \mathrm{ps}$, the left and right domains appear dark, because $\vec{M}$ has rotated in the opposite direction. A second oscillation is indicated by the snapshot at $t=$ 1400 ps. Micromagnetic simulations yield very similar oscillations of the magnetization, even though the exact pattern has not been reproduced.

In our experiment, the predominant excitation mode is a precession of the magnetization in the two large domains. It is excited by the external field pulse directed perpendicular to $\vec{M}$ in these domains. Our observation of two pronounced maxima of the magnetization component parallel to the field confirms the dynamical motion of $\vec{M}$. In our case, the excitation field can be described as an oscillating field with considerable contribution of overtones rather than a field pulse. Thus, the system resembles a

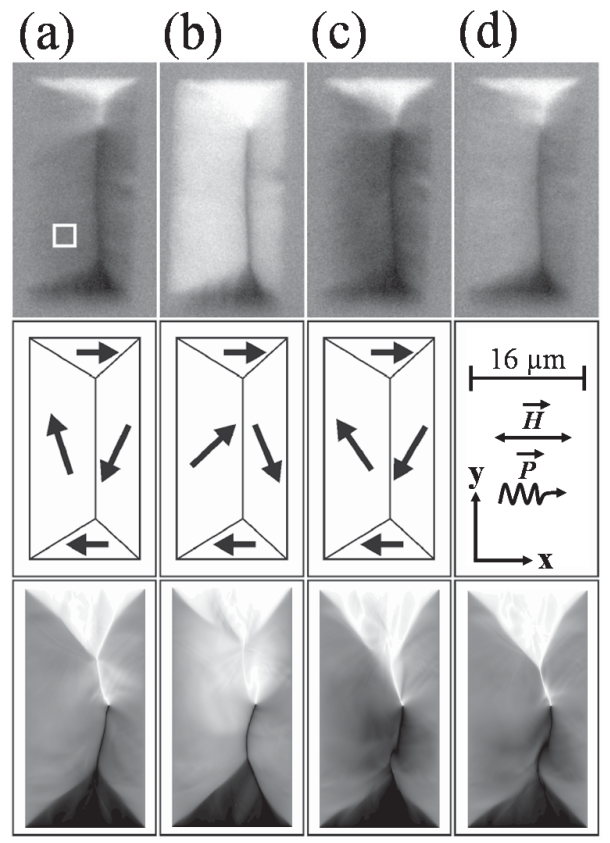

FIG. 2. Selected XMCD images showing the time evolution of the $x$ component of the magnetization (bright areas are magnetized to the right, dark areas to the left) in a Permalloy platelet $(16 \mu \mathrm{m} \times 32 \mu \mathrm{m})$ for delay times $t=0 \mathrm{ps}$ (a), $600 \mathrm{ps}$ (b), $1100 \mathrm{ps}$ (c), and $1400 \mathrm{ps}$ (d). The orientation of the exciting ac field and the photon polarization $\vec{P}$ are in the $x$ direction. Sketches of the corresponding domain patterns are shown in the second row. In the third row, micromagnetic simulation results are shown for a Permalloy platelet with linearly reduced dimensions $(8 \mu \mathrm{m} \times 16 \mu \mathrm{m} \times 5 \mathrm{~nm}$, cell size $10 \mathrm{~nm})$. 
driven oscillator, and we observe the dynamical answer of the system to the periodic excitation instead of a damped oscillatory relaxation to equilibrium.

To determine the frequencies and amplitudes of the data shown in Figs. 2(a)-2(d), we analyze the rotation angle $\phi(t)= \pm \arccos \left(M_{x} / M\right)$ by averaging over the boxes shown in Fig. 2(a). The intensity of the small closure domains was taken as a reference, since no initial torque acts on the magnetization within these domains. The rotation angle shows pronounced extrema at $t=600,1100$, 1450, and 1900 ps in the left domain [Fig. 3(a)]. A rough estimate of the eigenfrequency can be made from the second oscillation of $\vec{M}$ (indicated by the period $T$ ). In this case, the least altering of the eigenfrequency can be expected. For the left domain, we thus estimate a value of $1.25 \mathrm{GHz}$.

The domain walls and, particularly, the $180^{\circ}$-Néel wall move only very little throughout the series shown in Figs. 2(a)-2(d). Close to the maximum of the field pulse at $t=600 \mathrm{ps}$, the upper triangular domain $[\vec{M} \| \vec{H}(t)]$ grows while the lower black domain shrinks. This occurs through a bulging of the $90^{\circ}$ domain walls downwards. This bulging behavior is similar to an observation in square particles [1]. The wall velocity [Fig. 3(b)] does not exceed $10^{3} \mathrm{~m} \mathrm{~s}^{-1}$ in agreement with Ref. [1]. The $180^{\circ}$-Néel wall movement is in close relation to the oscillation of the magnetization in the left and right domains. We do not observe a significant movement of the vortex. This is not a contradiction to previous observations of a vortex motion perpendicular [1] or parallel to the applied field [3], because in our case the excitation frequency is too high for the slow velocity of the vortex.

The most interesting phenomenon is the mean shift of the $180^{\circ}$-Néel wall out of its symmetrical position to the right [see Figs. 4(b)-4(d)]. This shift increases with the

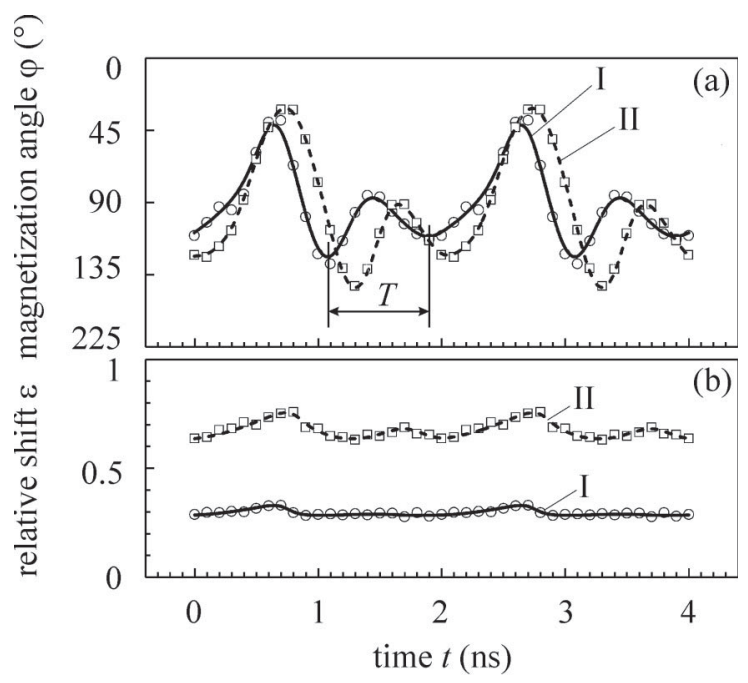

FIG. 3. (a) Time evolution of $M_{x}$ averaged over the box shown in Fig. 2(a) for field amplitudes I and II. Two periods are shown. (b) Displacement $\epsilon=2 \Delta x / w$ of the $180^{\circ}$-Néel wall parallel to $H_{x}(t)$. amplitude of the exciting field. This shift cannot be caused by the external field directly, because the field is directed parallel to the magnetization in the domain wall and, thus, causes no torque. Moreover, the field is oscillating and the mean field averaged over one cycle disappears. Of course, a movement of the wall could be induced by a magnetic field along the $y$ axis. Since the domain walls can move freely in our low-anisotropy sample, the walls will adjust in a quasistatic external field such that the sum of the demagnetizing field $H_{d}$ and the external field is zero. $H_{d}=N_{y} M \epsilon$ is given by the magnetic moment $m=M V \epsilon$ of the particle $\left(\epsilon=2 \Delta x / w\right.$, denoting the relative shift of the $180^{\circ}$ domain wall) and the demagnetizing factor $N_{y} \approx t / l$ (thickness $t$ and length $l$ ) roughly approximated assuming an ellipsoidal particle. The required external field for a domain wall shift to the right edge $(\epsilon=1)$ is 3 Oe, i.e., on the same order of magnitude as the field amplitude. Such a large field component can certainly not be caused by a misalignment of the particle and the waveguide or by stray fields from the leads. The presence of static fields can also be excluded (the vacuum chamber itself is made out of $\mu$ metal in order to shield external fields fields), as the shift of the domain wall would then be independent of the exciting field. However, Fig. 4 clearly shows that $\epsilon$ increases with the field amplitude. For the largest field amplitudes applied in this experiment, the platelet is nearly saturated with the magnetization vector pointing upwards [Fig. 4(d)].

The observed effect can be explained only by the following dynamic response of the system on the periodic excitation: As a general physical principle, a system with a continuous source (exciting field) and sink (spin damping) of power assumes a state with maximum energy stored in the system and, thus, maximizes the entropy production.

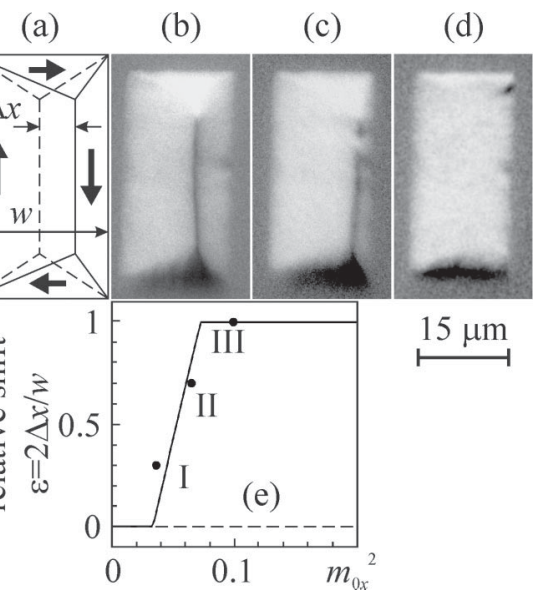

FIG. 4. Snapshots of the Permalloy platelet with the domain pattern sketched in (a) at the time $t=600 \mathrm{ps}$ excited with three different amplitudes [see Fig. 1(a)] denoted as I (b), II (c), and III (d). In (c), the domain wall comprises additional vortices appearing aperiodically, in some cases at higher amplitudes. A comparison of the numerical solution obtained from Eq. (3) and the experimentally determined mean domain wall shift $\epsilon$ is shown in (e). 
The energy stored in our particle is given mainly by the magnetization precession in the large domains. The system is excited with a significant oscillating field component of $1 \mathrm{GHz}$, i.e., just below the resonance frequency of the freerunning system. If the domain wall is shifted to the right, the effective field determining the precession frequency and, consequently, the resonance frequency will be reduced in the left domain and vice versa in the right domain. As a consequence, the amplitude of the precession will increase in the left domain and decrease in the right domain. Since the precession energy is proportional to the square of the amplitude, the total energy has increased. Moreover, the domain with the larger amplitude has grown, which also helps to increase the stored energy. The stored precession energy is finally balanced by the stray field energy, because of the resulting finite magnetic moment of the particle. The initial domain wall movement can occur to the left or to the right. However, we observed exclusively a shift to the right. Small inhomogeneities or a small vortex motion as described in Ref. [3] could be the reason.

In order to substantiate this qualitative picture, we estimate the contributing energies in the following. The amplitude of a forced oscillator with small damping is given by $A(\xi)=C\left(1-\xi^{2}\right)^{-1}$ at the exciting frequency $\xi=$ $\omega / \omega_{0}$ normalized to the resonance frequency $\omega_{0}$. If $\omega_{0}$ is varied, the amplitude will change by:

$$
\beta=\frac{A^{\prime}(\xi)}{A(\xi)} \Delta \omega_{0}=-\frac{2 \xi^{2}}{1-\xi^{2}} \frac{\Delta \omega_{0}}{\omega_{0}} .
$$

The resonance frequency $\omega_{0}$ of the two large domains can be derived from the Landau-Lifshitz-Gilbert equation: $\omega_{0}(\epsilon)=\gamma \mu_{0} M \sqrt{N_{x}-N_{y}^{-}} \approx \gamma \mu_{0} M \sqrt{\pi t / w(1+\epsilon)}$, assuming demagnetization factors $N_{i}$ for an ellipsoidal particle of similar dimensions as the magnetic domains. The resonance frequency decreases with increasing shift $\epsilon=$ $2 \Delta x / w$ of the domain wall: $\Delta \omega_{0} / \omega_{0}(0)=\left[(1+\epsilon)^{-1 / 2}-\right.$ $1]$. We calculate the change of the precession energy when the domain wall is shifted by $\epsilon$ as

$$
\Delta E_{p} / E_{p}=\beta^{2}+2 \beta \epsilon,
$$

where $E_{p}$ denotes the total precession energy of the excited mode. Using the experimentally accessible amplitude of the magnetization component $M_{x}=M m_{x}$ along the $x$ axis and the demagnetization factor $N_{x}$, we can express the energy as $E_{p}=\frac{1}{2} V \mu_{0} N_{x} M^{2} m_{x}^{2}$. The stray field energy $\Delta E_{s}$ balancing $\Delta E_{p}$ also increases with increasing domain wall shift: $\Delta E_{s}=\frac{1}{2} V \mu_{0} N_{y} M^{2} \epsilon^{2}$. From the equilibrium condition $\Delta E_{p}=\Delta E_{s}$, one obtains the implicit equation:

$$
\left(N_{x} / N_{y}\right)\left(\beta^{2}+2 \beta \epsilon\right) m_{x}^{2}=\epsilon^{2},
$$

determining the shift $\epsilon$ as a function of the oscillation amplitude $m_{x}$. If $m_{x}$ exceeds a critical value, a nontrivial solution $\epsilon \neq 0$ exists. As expected, $\epsilon$ increases with increasing amplitude of the oscillation. A comparison of $\epsilon\left(m_{x}\right)$ obtained from Eq. (3) to the experimental data is shown in Fig. 4(e) with the parameter $2 \xi^{2} /\left(1-\xi^{2}\right)=3.8$ corresponding to a relative exciting frequency $\xi=0.81$, i.e., $\omega / 2 \pi \approx 1 \mathrm{GHz}$. Even though we performed a rough approximation of the contributing energies, the experimental data can be described by our model quite well, thus confirming our assumptions. Micromagnetic simulations confirm the basic assumptions of this model, as will be discussed in more detail in a forthcoming publication.

In conclusion, we found an induced magnetic moment in a rectangular platelet oriented perpendicular to an exciting oscillatory magnetic field. This phenomenon can be explained by a self-trapping spin-wave mode. When the system is excited just below the resonance frequency, the magnetization distribution adapts itself to gain more energy out of the exciting field. In the dynamically steady state of feeding constantly energy into the platelet by the exciting field that finally dissipates into the heat bath by magnon-phonon interaction, the increase of entropy is thus maximized. Above a threshold, the near-resonance spinwave mode thus causes an effective force perpendicular to the $180^{\circ}$-Néel wall in the center of the particle that is balanced by the restoring force due to the stray field energy.

This work was supported by the DFG (priority program "magnetization dynamics") and BMBF (03N6500). Thanks are due to the BESSY staff and, in particular, H. Schmitz (HMI, Berlin) for excellent support and I. Mönch and J. Vinzelberg (IFW Dresden) for sample preparation. M. B. thanks G. Meier (Universität Hamburg) for fruitful discussions and insights and the DFG (Sonderforschungsbereich 508 "Quantenmaterialien") for financial support.

*Electronic address: elmers@uni-mainz.de

[1] J. Raabe et al., Phys. Rev. Lett. 94, 217204 (2005).

[2] K. Perzlmaier et al., Phys. Rev. Lett. 94, 057202 (2005).

[3] S.-B. Choe et al., Science 304, 420 (2004).

[4] H. Stoll et al., Appl. Phys. Lett. 84, 3328 (2004).

[5] J. P. Park et al., Phys. Rev. B 67, 020403(R) (2003).

[6] S. O. Demokritov et al., Phys. Rep. 348, 441 (2001).

[7] T. M. Crawford et al., Phys. Rev. B 67, 024411 (2003).

[8] See, for example, A. Hubert and R. Schäfer, Magnetic Domains (Springer, Berlin, 1998).

[9] L. Landau et al., Phys. Z. Sowjetunion 8, 153 (1935).

[10] C. M. Schneider et al., Appl. Phys. Lett. 85, 2562 (2004).

[11] C. M. Schneider et al., Rep. Prog. Phys. 65, 1785(R) (2002).

[12] D. Neeb et al., J. Phys. Condens. Matter 17, S1381 (2005).

[13] J. Feikes et al., in Proceedings of the 9th European Particle Accelerator Conference (EPAC 2004), Lucern, Switzerland (European Physical Society Accelerator Group, Lucern, Switzerland, 2004), p. 1954; www. BESSY.de.

[14] http://math.nist.gov/oommf/. For the simulations, standard Permalloy parameters $\left(A=1.3 \times 10^{-12} \mathrm{~J} / \mathrm{m}, \quad M_{s}=\right.$ $\left.8.6 \times 10^{5} \mathrm{kA} / \mathrm{m}, \alpha=0.005\right)$ were used. 